\title{
Rounds in Combinatorial Search
}

\author{
Extended abstract
}

\author{
Gábor Wiener ${ }^{1}$ \\ Budapest University of Technology and Economics, \\ Department of Computer Science and Information Theory \\ H-1521, Budapest, Hungary \\ wiener@cs.bme.hu
}

\begin{abstract}
The search complexity of a separating system $\mathcal{H} \subseteq 2^{[m]}$ is the minimum number of questions of type " $x \in H$ ?" (where $H \in \mathcal{H}$ ) needed in the worst case to determine a hidden element $x \in[\mathrm{m}]$. If we are allowed to ask the questions in at most $k$ batches then we speak of the $k$-round (or $k$-stage) complexity of $\mathcal{H}$, denoted by $\mathrm{c}_{k}(\mathcal{H})$. While 1 -round and $m$-round complexities (called non-adaptive and adaptive complexities, respectively) are widely studied (see for example Aigner [1]), much less is known about other possible values of $k$, though the cases with small values of $k$ (tipically $k=2$ ) attracted significant attention recently, due to their applications in DNA library screening. It is clear that $|\mathcal{H}| \geq \mathrm{c}_{1}(\mathcal{H}) \geq \mathrm{c}_{2}(\mathcal{H}) \geq \ldots \geq \mathrm{c}_{m}(\mathcal{H})$. A group of problems raised by $\mathrm{G}$. O. H. Katona [6] is to characterize those separating systems for which some of these inequalities are tight. In this paper we are discussing set systems $\mathcal{H}$ with the property $|\mathcal{H}|=\mathrm{c}_{k}(\mathcal{H})$ for any $k \geq 3$. We give a necessary condition for this property by proving a theorem about traces of hypergraphs which also has its own interest.
\end{abstract}

Keywords. Search, group testing, adaptiveness, hypergraph, trace

\section{Preliminaries}

We denote the set of the first $m$ positive integers by $[m]$. A set system $\mathcal{A} \subseteq 2^{[m]}$ is said to be a separating system if for any pair of distinct elements $x, y \in[m]$ there exists a set in $\mathcal{A}$ that contains exactly one of them. A separating system $\mathcal{A}$ is minimal if no $\mathcal{B} \subset \mathcal{A}$ is separating.

A hypergraph is a pair $(V, \mathcal{E})$, where $V$ is a finite set, called the vertices of the hypergraph and $\mathcal{E}$ is a collection of subsets of $V$, called the (hyper)edges of the hypergraph. Notice that $\mathcal{E}$ is not necessarily a set, that is, hyperedges may have multiplicity greater than 1 . If every hyperedge has multiplicity 1 then the hypergraph is called simple. It is obvious that edge sets of simple hypergraphs and set systems are the same. If the restriction of a simple hypergraph to any proper subset of the vertices is not simple then we speak of a minimal simple hypergraph. The set of all minimal simple hypergraphs on the vertex set $[n]$ having $m$ hyperedges is denoted by $M S H(n, m)$. The multiplicity of a set of

Dagstuhl Seminar Proceedings 09281

Search Methodologies

http://drops.dagstuhl.de/opus/volltexte/2009/2239 
vertices $X$ in a hypergraph $\mathcal{H}$ is the number of occurences of $X$ as an edge and is denoted by $m_{\mathcal{H}}(X)$. Sometimes, if it does not cause any misunderstanding we identify hypergraphs by their edge set.

Let $\mathcal{H}=(V, \mathcal{E})$ be a hypergraph and consider any linear order of $V$ and $\mathcal{E}$. The incidence matrix of $\mathcal{H}$ is a $0-1$ matrix $M_{\mathcal{H}}=\left(m_{i j}\right)_{|\mathcal{E}|,|V|}$, where $m_{i j}$ is 1 if and only if the $i^{t h}$ edge contains the $j^{\text {th }}$ vertex. The incidence matrix of a set system $\mathcal{A} \subseteq 2^{S}$ is defined as the incidence matrix of the simple hypergraph having vertex set $S$ and edge set $\mathcal{A}$ and is denoted by $M_{\mathcal{A}}$. It is obvious that any row and column permutation of an incidence matrix of a hypergraph (set system) is also an incidence matrix of the same hypergraph (set system) and that any 0-1 matrix is an incidence matrix of some hypergraph. The dual of a hypergraph $\mathcal{H}$ is the hypergraph $\mathcal{H}^{*}$ whose incidence matrix is $M_{\mathcal{H}}^{T}$. The dual of a set system $\mathcal{A}$ is the collection of edges of the hypergraph whose incidence matrix is $M_{\mathcal{A}}^{T}$. Note that $\mathcal{A}^{*}$ is not necessarily a set system.

It is obvious that a hypergraph $\mathcal{H}$ is simple if and only if $M_{\mathcal{H}}$ has no identical rows and that a set system $\mathcal{A}$ is separating if and only if $M_{\mathcal{A}}$ contains no identical columns.

A set system $\mathcal{A}$ of cardinality $k+1$ is called a $k$-star if it contains a set $A$ such that for any $B \in \mathcal{A}, B \neq A: A \subseteq B$ and $|B \backslash A|=1$.

A set system $\mathcal{A} \subseteq 2^{[m]}$ is said to be hereditary if $A \in \mathcal{A}$ and $B \subseteq A$ implies $B \in \mathcal{A}$.

A set system $\mathcal{A} \subseteq 2^{[m]}$ is said to be a representation of a set system $\mathcal{B} \subseteq 2^{[m]}$ if there exist a linear order of the sets of $\mathcal{A}\left(A_{1}, A_{2}, \ldots, A_{r}\right)$ and $\mathcal{B}\left(B_{1}, B_{2}, \ldots, B_{r}\right)$ and a permutation $\pi$ of the elements of $[m]$, such that for any $i \leq r=|\mathcal{B}|$ we have either $A_{i}=\left\{\pi(j): j \in B_{i}\right\}$ or $A_{i}=\left\{\pi(j): j \notin B_{i}\right\}$. In other words, $\mathcal{A}$ is a representation of $\mathcal{B}$ if they have the same cardinality and their incidence matrices can be transformed to each other by row and column permutations and by complementing some rows (but not columns), where complementing a row means that we change the 1 entries of the row to 0 entries and vice versa.

\section{Introduction}

Let $\mathcal{H} \subseteq 2^{[m]}$ be an arbitrary separating system (called the question sets) and $x \in[m]$ an unknown element. Our aim is to find $x$ by asking questions of type "x $\in H$ ?", where $H \in \mathcal{H}$. A sequence of questions is called a search algorithm (or shortly an algorithm) if given the answers we can determine $x$ uniquely.

An algorithm is said to be adaptive (or dynamic) if the choice of a question set may depend on the values obtained until then. If the questions are all fixed beforehand then we speak of a non-adaptive (or static) algorithm. More generally, if we are allowed to ask the questions in at most $k$ batches (that is, we ask some questions, receive the answers, ask again some questions, receive the answers, and so on, at most $k$ times) then we speak of a $k$-round (or $k$-stage) algorithm.

The length of an algorithm $\mathbf{A}$ for the element $x$, denoted by $l_{x}(\mathbf{A})$ is $l$ if the sequence contains $l$ questions and the first $l-1$ answers does not determine $x$ 
uniquely. The (worst case) cost of an algorithm $\mathbf{A}$ is $g(\mathbf{A})=\max _{x \in[m]} l_{x}(\mathbf{A})$. The adaptive (search) complexity of the set system $\mathcal{H}$ is $\mathrm{c}(\mathcal{H})=\min g(\mathbf{A})$ considering all adaptive algorithms $\mathbf{A}$. The non-adaptive, and $k$-round complexities are defined similarly and are denoted by $c_{n a}(\mathcal{H})$ and $c_{k}(\mathcal{H})$, respectively. Notice that since $\mathcal{H}$ is separating, these definitions are correct. For a detailed treatment of adaptive and non-adaptive search the reader is referred to the book by Aigner [1]. Much less is known about $k$-round search for arbitrary values of $k$, though the cases with small values of $k$ (tipically $k=2$ ) attracted significant attention recently, due to their applications in DNA library screening.

It is obvious that

$$
|\mathcal{H}| \geq \mathrm{c}_{n a}(\mathcal{H})=\mathrm{c}_{1}(\mathcal{H}) \geq \mathrm{c}_{2}(\mathcal{H}) \geq \ldots \geq \mathrm{c}_{m}(\mathcal{H})=\mathrm{c}(\mathcal{H})
$$

A problem raised by G. O. H. Katona [6] is to characterize those separating systems $\mathcal{H} \subseteq 2^{[m]}$ for which certain inequalities of (1) are tight. In the present paper our aim is to examine separating systems $\mathcal{H} \subseteq 2^{[m]}$ with the property $|\mathcal{H}|=\mathrm{c}_{k}(\mathcal{H})$ for any $k \geq 3$.

More precisely, we will show that if $\mathrm{c}_{k}(\mathcal{H})=|\mathcal{H}|$ for some $k \geq 3$ then the dual of $\mathcal{H}$ contains a $\left\lceil\frac{n^{2}}{2 m-n-2}\right\rceil$-star, where $n=|\mathcal{H}|$.

\section{Results}

We would like to examine separating systems $\mathcal{H} \subseteq 2^{[m]}$ for which $\mathrm{c}_{k}(\mathcal{H})=|\mathcal{H}|$ for some $k \geq 3$. This condition implies $\mathrm{c}(\mathcal{H})=|\mathcal{H}|$, from which $|\mathcal{H}| \leq m-1$ follows easily. It is more interesting that even $c_{1}(\mathcal{H})=|\mathcal{H}|$ implies $|\mathcal{H}| \leq m-1$, in other words, a minimal separating system $\mathcal{H} \subseteq 2^{[m]}$ contains at most $m-1$ sets, as it was first observed by Bondy [4]. Notice that both results are sharp, just consider $\mathcal{H}=\{\{1\},\{2\}, \ldots,\{m-1\}\}$.

Using Bondy's result it is not difficult to characterize those systems whose $k$-round complexity is $m-1$ for any $k \geq 2$.

Lemma 1. Let $k \geq 2$. For a separating system $\mathcal{H} \subseteq 2^{[m]}, c_{k}(\mathcal{H})=m-1$ if and only if $\mathcal{M}=\{\{1\},\{2\}, \ldots,\{m-1\}\}$ is a representation of $\mathcal{H}$.

The main theorem of this paper is the following.

Theorem 1. Let $\mathcal{H} \subseteq 2^{[m]}$ be a separating system for which $c_{k}(\mathcal{H})=|\mathcal{H}|$ for some $k \geq 3$ and let $n=|\mathcal{H}|$. Then $\mathcal{H}^{*}$ contains $a\left\lceil\frac{n^{2}}{2 m-n-2}\right\rceil$-star.

Proof. If for some $k \geq 3$ we have $\mathrm{c}_{k}(\mathcal{H})=|\mathcal{H}|$ then $\mathrm{c}_{3}(\mathcal{H})=|\mathcal{H}|$. We show that this implies that $\mathcal{H}^{*}$ contains a $\left\lceil\frac{n^{2}}{2 m-n-2}\right\rceil$-star.

The proof is based on the following theorem about hypergraphs.

Theorem 2. Let $\mathcal{A} \in M S H(n, m)$. Then there exists a subset $X \subseteq[n]$ of cardinality $\left\lceil\frac{n^{2}}{2 m-n-2}\right\rceil$, such that deleting $X$ we obtain a hypergraph where every hyperedge has multiplicity at most $\left\lceil\frac{n^{2}}{2 m-n-2}\right\rceil+1$. 
The sketch of the proof of Theorem 2 can be found in Section 4 .

Let us denote the number $\left\lceil\frac{n^{2}}{2 m-n-2}\right\rceil$ by $r$. Consider now the set system $\mathcal{H}^{*}$. Since $\mathcal{H}$ is separating, $\mathcal{H}^{*}$ is also a set system (that is, it contains distinct sets), in other words it is the hyperedge set of a simple hypergraph $\mathcal{G}$ on the vertices corresponding to the sets of $\mathcal{H}$. Observe now that $\mathrm{c}_{n a}(\mathcal{H})=|\mathcal{H}|$ (because $\left.\mathrm{c}_{3}(\mathcal{H})=|\mathcal{H}|\right)$, so $\mathcal{H}$ is a minimal separating system, thus $\mathcal{G}$ is a minimal simple hypergraph having $n$ vertices and $m$ hyperedges. Now applying Theorem 2 for $\mathcal{G}$ we see that there exists a subset of the vertices $X,|X|=r$, such that deleting $X$ we obtain a hypergraph where every hyperedge has multiplicity at most $r+1$. This subset $X$ of the vertices of $\mathcal{H}^{*}$ correspond to a subset $\mathcal{X}$ of the original set system $\mathcal{H}$. Considering the incidence matrix of $\mathcal{H}$ one can see that deleting the rows corresponding to $\mathcal{X}$ we obtain a matrix where every column appears at most $r+1$ times.

Suppose now that we ask the sets of $\mathcal{H} \backslash \mathcal{X}$ in the first round of a 3-round search algorithm. Given the answers we know that the unknown element is one from a set $Y \subseteq[m]$, where $|Y| \leq r+1$, because no column appears more than $r+1$ times in the incidence matrix of $\mathcal{H} \backslash \mathcal{X}$.

Since $c_{3}(\mathcal{H})=|\mathcal{H}|$, we have to ask all the remaining sets of $\mathcal{H}$ in two more rounds to determine the hidden element. That is, we have to ask $|\mathcal{X}|=r$ sets in two rounds to find an element in $Y$, which has at most $r+1$ elements. By Lemma 1 this is possible if and only if $Y=r+1$ and the restriction of $\mathcal{X}$ to $Y$ contains only one-element sets. In other words, the incidence matrix of the restriction of $\mathcal{X}$ to $Y$ is an $r \times r$ identity matrix plus an all-zero column. Since for the elements of $Y$ we received the same answers in the first round, these elements form an $r$-star in $\mathcal{H}^{*}$.

\section{Sketch of proof of Theorem 2}

Let $\mathcal{H}$ be a hypergraph on the vertex set $[n]$. Let us denote the hypergraph obtained from $\mathcal{H}$ by deleting a subset $X$ of the vertices (that is, taking the restriction of $\mathcal{H}$ to $\bar{X}=[n] \backslash X)$ by $\left.\mathcal{H}\right|_{\bar{X}}$. Recall that $m_{\mathcal{H}}(E)$ denotes the multiplicity of the hyperedge $E$ in the hypergraph $\mathcal{H}$.

The following lemma can be proved using the down-compression technique of Alon [2] and Frankl [5].

Lemma 2. The following two statements are equivalent.

1. For every $\mathcal{A} \in M S H(n, m)$ there exists a set $X \subseteq[n]$ of cardinality $r$, such that for any set $S \subseteq \bar{X}$ we have $m_{\left.\mathcal{A}\right|_{\bar{X}}}(S) \leq s$.

2. For every hereditary $\mathcal{A} \in M S H(n, m)$ there exists a set $X \subseteq[n]$ of cardinality $r$, such that for any set $S \subseteq \bar{X}$ we have $m_{\left.\mathcal{A}\right|_{\bar{X}}}(S) \leq s$.

By Lemma 2 we only have to prove that for a hereditary minimal simple hypergraph having $n$ vertices and $m$ hyperedges there exists a subset of the

vertices $X$ of cardinality $\left\lceil\frac{n^{2}}{2 m-n-2}\right\rceil$, such that deleting $X$ we obtain a hypergraph where every hyperedge has multiplicity at most $\left\lceil\frac{n^{2}}{2 m-n-2}\right\rceil+1$. 
Let $\mathcal{A}$ be such a hypergraph. Observe that every vertex $v$ is contained in some hyperedge, otherwise $\mathcal{A}$ would not be minimal, thus by the hereditary property all 1-element sets are hyperedges of $\mathcal{A}$.

This means that the number of hyperedges of $\mathcal{A}$ containing at least two elements is $m-n-1$ (since $\mathcal{A}$ contains $n$ 1-element hyperedges and also the empty set). Consider now the graph $G$ on the vertex set $[n]$ whose edges are the 2-element sets of $\mathcal{A}$. $G$ has $n$ vertices and at most $m-n-1$ edges, thus by a corollary of Turán's theorem [7], [3, p. 282.] it contains a stable set $X$ of size $\left\lceil\frac{n^{2}}{2(m-n-1)+n}\right\rceil=\left\lceil\frac{n^{2}}{2 m-n-2}\right\rceil$. We show that $m_{\left.\mathcal{A}\right|_{\bar{X}}}(S) \leq\left\lceil\frac{n^{2}}{2 m-n-2}\right\rceil+1$ for any $S \subseteq[n]$. Actually, it suffices to show that $m_{\left.\mathcal{A}\right|_{\bar{X}}}(\emptyset) \leq\left\lceil\frac{n^{2}}{2 m-n-2}\right\rceil+1$, since by the hereditary property of $\mathcal{A}$ we have $m_{\left.\mathcal{A}\right|_{\bar{X}}}(\emptyset) \geq m_{\left.\mathcal{A}\right|_{\bar{X}}}(S)$ for any $S \subseteq[n]$.

By definition, $m_{\left.\mathcal{A}\right|_{\bar{X}}}(\emptyset)=|\{A \in \mathcal{A}: A \subseteq X\}|=|\mathcal{A}|_{X} \mid$.

If $i, j \in X(i \neq j)$, then $\{i, j\} \notin \mathcal{A}$, since $X$ is stable in $G$. Furthermore, there is no hyperedge in $\mathcal{A}$ that contains both $i$ and $j$, because $\mathcal{A}$ is hereditary. Thus $\left.\mathcal{A}\right|_{X}$ does not contain sets of size greater than 1 , so the number of distinct sets in $\left.\mathcal{A}\right|_{X}$ is at most $|X|+1=\left\lceil\frac{n^{2}}{2 m-n-2}\right\rceil+1$.

\section{References}

1. M. Aigner. Combinatorial Search, John Wiley, Chichester and Teubner, Stuttgart, 1988

2. N. Alon. On the density of sets of vectors, Discrete Mathematics 46 (1983), 199 202.

3. C. Berge. Graphs and Hypergraphs, North Holland,

4. J. A. Bondy. Induced subsets, Journal of Combinatorial Theory, Series B 12 (1972), 201-202.

5. P. Frankl. On the trace of finite sets, Journal of Combinatorial Theory, Series A 34, (1983), 41-45.

6. G. O. H. Katona. Personal communication, 2004

7. P. Turán. On an extremal problem in graph theory, (in Hungarian) Mat. Fiz. Lapok (1941) 48, p. 436-452. 